\title{
Enhancement of the magnetic anisotropy of Co clusters by Au capping
}

\author{
F. Luis, ${ }^{\text {a) }}$ J. Bartolomé, F. Bartolomé, M. J. Martínez, and L. M. García \\ Instituto de Ciencia de Materiales de Aragón, CSIC-Universidad de Zaragoza, 50009 Zaragoza, Spain \\ F. Petroff and C. Deranlot \\ Unité Mixte de Physique CNRS/Thales, Route Départementale 128, 91767 Palaiseau Cedex, France, \\ and Université Paris-Sud, 91405 Orsay Cedex, France \\ F. Wilhelm and A. Rogalev \\ European Synchrotron Radiation Facility, B.P. 38042 Grenoble, France
}

(Presented on 3 November 2005; published online 26 April 2006)

\begin{abstract}
We study the magnetic properties of Co nanoparticles, prepared by sputtering, with diameters ranging from 1 to $3.5 \mathrm{~nm}$. The effective anisotropy, which determines the activation energy for the magnetization reversal, increases with decreasing particle's size, showing the dominant role played by surface atoms. We find that the superparamagnetic blocking temperature and the coercive field are significatively enhanced when the clusters are capped by a thin Au layer. This enhancement is probably caused by the bonding or hybridization of $\mathrm{Co}$ and $\mathrm{Au}$ atoms at the interface between the two metals. It provides thus a method to modify the magnetic anisotropy, which can be of interest for the applications of magnetic nanoparticles. (C) 2006 American Institute of Physics.
\end{abstract}

[DOI: $10.1063 / 1.2172217$ ]

The magnetic anisotropy of magnetic nanoparticles is a key parameter for the application of these materials in data recording. ${ }^{1}$ The product of the anisotropy constant $K$ times the volume of the particle $V$ gives the activation energy $U$ required to reverse the particle's magnetization. At any temperature, the time stability of a magnetic bit against thermal fluctuations is determined by the Arrhenius law, ${ }^{2}$

$$
\tau=\tau_{0} \exp \left(\frac{U}{k_{\mathrm{B}} T}\right),
$$

where $\tau_{0}$ is an attempt time. Therefore, reducing the size of magnetic bits down to the nanometer range requires new ways of enhancing their anisotropy.

In the case of nanometer-sized particles, the anisotropy usually exceeds the magnetocrystalline anisotropy of the bulk material. ${ }^{3-7}$ It is then dominated by size-dependent effects arising from shape, stress, and their large surface-tovolume ratio. The lower symmetry of surface atomic sites leads to an enhanced anisotropy. This phenomenon is reminiscent of the surface anisotropy observed in thin magnetic films. ${ }^{8,9}$ Interestingly, the anisotropy of thin films depends not only on the layer's thickness but also on the nature of the substrate on which the films grow. ${ }^{9,10}$ This effect is associated to the interaction or bonding of surface magnetic atoms to those of the substrate or a capping layer. ${ }^{11,12}$ Here, we show that capping Co clusters by a thin noble metal layer provides also a relatively simple method to enhance their anisotropy.

Nanometer-sized Co clusters were prepared, as described previously, ${ }^{13}$ by sequential sputtering of amorphous alumina and Co layers on a Si substrate. The Co layer segregates into nearly spherical metallic clusters, which within each layer is

\footnotetext{
a) Author to whom correspondence should be addressed; electronic mail: fluis@unizar.es
}

separated by an average distance of $2.2 \mathrm{~nm} .{ }^{13}$ Their size can be controlled by varying the nominal thickness $t_{\mathrm{Co}}$ that the Co film would have if it were continuous. Below $t_{\mathrm{Co}}=1 \mathrm{~nm}$, clusters crystallize with the fcc Co phase. ${ }^{13}$ In this work, we studied granular films with $t_{\mathrm{Al}_{2} \mathrm{O}_{3}}=3$ and $0.2 \mathrm{~nm} \leqslant t_{\mathrm{Co}}$ $\leqslant 0.7 \mathrm{~nm}$, corresponding to the average particle diameters $D$ between 1 and $3.5 \mathrm{~nm}$. Gold-capped clusters were prepared by depositing a thin $(1.5 \mathrm{~nm})$ Au film right after the deposition of the Co clusters. Therefore, we do not expect the crystal structure and morphology of the clusters to be affected by the capping, as it is indeed suggested by our magnetic measurements (see below). Usually samples are made by piling up a number $N$ of $\mathrm{Al}_{2} \mathrm{O}_{3} / \mathrm{Co}$ or $\mathrm{Al}_{2} \mathrm{O}_{3} / \mathrm{Co} / \mathrm{Au}$ repetition units.

Magnetization and ac susceptibility experiments were performed in a commercial superconducting quantum interference device (SQUID) magnetometer. The applied magnetic field was always parallel to the plane of the samples to minimize demagnetization effects. The diamagnetic contribution of Si was determined by measuring a bare substrate and then subtracted from all magnetic data. The samples contain also a fraction of isolated atoms or very small clusters, which do not form particles. Their magnetic susceptibility can be estimated from high-field (5 T) magnetization measurements performed down to low temperatures $(T>2 \mathrm{~K}){ }^{6}$ It is negligible with respect to the susceptibility of the particles in the whole temperature range of interest here $(5 \mathrm{~K}$ $\leqslant T \leqslant 100 \mathrm{~K}$ ). In addition to these magnetic studies, we also performed $\mathrm{x}$-ray magnetic circular dichroism (XMCD) experiments at the $L_{2,3}$ absorption edges of Au. These measurements were done at the ID12 beam line of the ESRF in Grenoble.

The dc magnetic susceptibility $\chi$ and magnetization isotherms $M$ of uncapped and Au-capped Co clusters with $D$ $\simeq 1.8 \mathrm{~nm}$ are plotted in Figs. 1 and 2 . The equilibrium mag- 


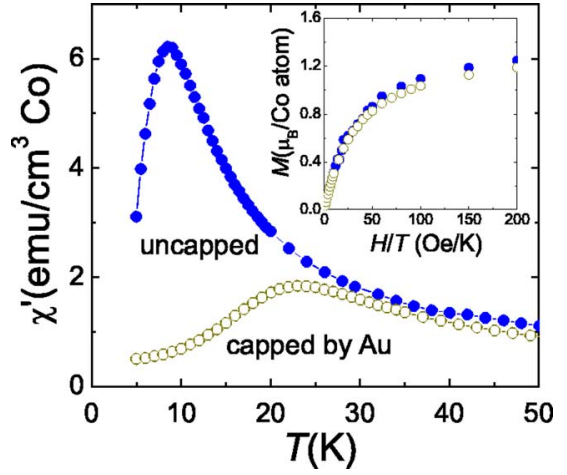

FIG. 1. (Color online) dc susceptibility $\chi$ measured after cooling the samples in zero field of two granular Co multilayers with $t_{\mathrm{Co}}=0.4 \mathrm{~nm}$ and $N=25$ layers: $(\bigcirc)$ uncapped clusters; $(\bigcirc)$ clusters capped by a $1.5 \mathrm{~nm}$ thick Au layer. The inset shows the magnetization curves of the same samples measured at $100 \mathrm{~K}$.

netic response of the nanoparticles, measured above the superparamagnetic blocking temperature $T_{b}$ where $\tau$ is much shorter than the typical experimental time scales $\left(t_{\exp }\right.$ $\sim 140 \mathrm{~s}$ ), is almost the same for both samples. This shows that capping with Au does not modify much of the particle's size distribution. By contrast, the temperature of the susceptibility maximum increases by almost a factor 3 , from $8.5 \mathrm{~K}$ for the uncapped clusters up to $23.5 \mathrm{~K}$ for clusters capped by Au. As follows from the Arrhenius law, Eq. (1), this blocking temperature reflects the freezing of the magnetic moments that takes place when $\tau$ becomes of the order of $t_{\text {exp }}$. These data suggest therefore that the magnetization reversal of capped clusters is slower than that of clusters in alumina, i.e., the magnetization becomes more stable against thermal fluctuations. The same qualitative conclusion can be drawn from hysteresis loops measured in the blocked temperature region $\left(T \ll T_{b}\right)$ that are plotted in Fig. 2. Capping with Au leads to a six times larger coercive field (from $H_{c}=0.5-3.15 \mathrm{kOe}$ ), in other words, it makes the clusters magnetically harder.

In previous studies of samples prepared by the same method, we used ac susceptibility data to obtain the magnetic anisotropy. ${ }^{6}$ Here, we apply the same technique to the uncapped and capped Co particles. As shown in Fig. 3, imaginary susceptibility data $\chi^{\prime \prime}$ measured at different frequencies, $0.1 \mathrm{~Hz} \leqslant \omega / 2 \pi \leqslant 90 \mathrm{~Hz}$, fall in a single curve when they are plotted against the scaling variable $T \ln \left(1 / \omega \tau_{0}\right)$. The prefactor $\tau_{0} \sim 10^{-15} \mathrm{~s}$ is estimated a priori from the Arrhenius plot

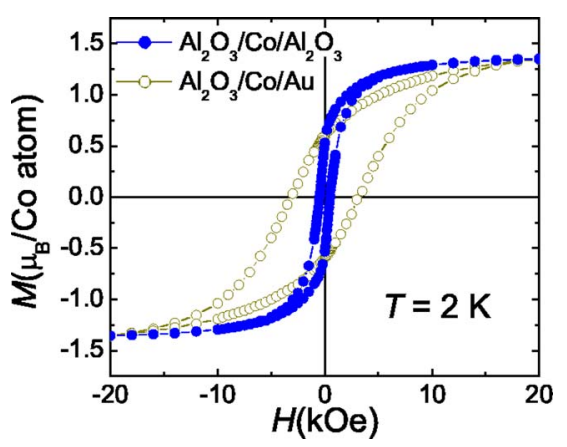

FIG. 2. (Color online) Magnetization hysteresis loops of two samples of capped and uncapped Co clusters with $t_{\mathrm{Co}}=0.4 \mathrm{~nm}$ and $N=25$ measured at $2 \mathrm{~K}$.

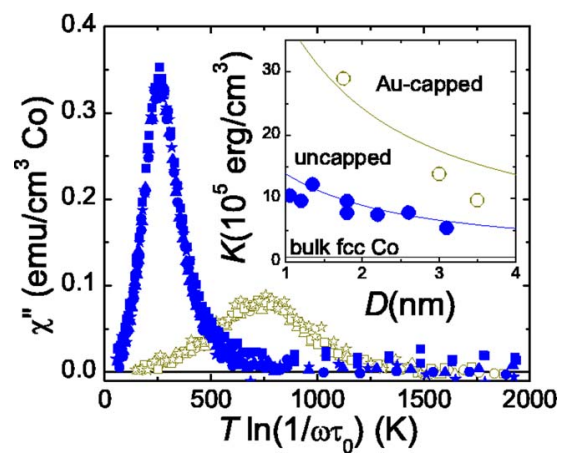

FIG. 3. (Color online) Scaling plot of the imaginary susceptibility $\chi^{\prime \prime}$ measured at $\omega / 2 \pi=0.1 \mathrm{~Hz}$ (circles), $1 \mathrm{~Hz}$ (triangles), $10 \mathrm{~Hz}$ (squares), and $90 \mathrm{~Hz}$ (stars) for two samples of Co clusters with $t_{\mathrm{Co}}=0.4 \mathrm{~nm}$ and $N=25$. Solid symbols: uncapped clusters; open symbols: Au-capped clusters. The inset shows the enhancement of the size-dependent magnetic anisotropy constant $K$ caused by Au capping. The solid lines are least-squares fits to a core-shell model [Eq. (2)].

of the frequency-dependent blocking temperature. The scaling plot is directly related to the distribution of activation energies $U$. We see that, for a given average diameter, capping by Au leads to a remarkably large shift of the distribution towards larger $U$ values.

Once we have the distribution of particle's sizes and of activation energies, the effective anisotropy constant $K$ can be estimated simply as the ratio between $U$ and $V$ for particles with average diameter $D$ (see Ref. 6 for details). We find (see the inset of Fig. 3) that $K$ is much larger than the anisotropy of bulk fcc Co (Ref. 14) $K_{\text {bulk }} \sim 7 \times 10^{5}$ and that it increases with decreasing size. It is interesting to mention that the value $K \simeq 8 \times 10^{6} \mathrm{erg} / \mathrm{cm}^{3}$ we obtain for $D$ $\simeq 2.6 \mathrm{~nm}(N \sim 800$ atoms per cluster $)$ is of the same order as the uniaxial anisotropy constant obtained from first principles calculations for Co clusters of about 600 atoms. $^{15}$ These results indicate that surface atoms are much more effective as a source of anisotropy than core atoms. In addition, Au-capped clusters show, for all sizes, a much stronger anisotropy than clusters embedded in alumina.

We can understand the size-dependent $K$ using a very simple picture in which the surface and core atoms have different anisotropy constants, $K_{\text {surface }}$ and $K_{\text {bulk }}$, respectively. Within this model, $K$ can be fitted by the following phenomenological expression:

$$
K=f K_{\text {surface }}+(1-f) K_{\text {bulk }},
$$

where $f \simeq 1-(1-0.4 / D)^{3}$ is the fraction of surface atoms in a fcc cluster. We find that $K_{\text {surface }}=25 K_{\text {bulk }}$ when the clusters are embedded in alumina, whereas capping with Au makes it almost three times larger $\left(K_{\text {surface }} \simeq 70 K_{\text {bulk }}\right)$.

The dependence of the surface anisotropy on the surrounding material is strongly reminiscent of the behavior observed for thin films of Co. ${ }^{9,10}$ For $\mathrm{Au} / \mathrm{Co} / \mathrm{Au}$ films the anisotropy gives rise to a preferred perpendicular magnetization for less than 11 atomic layers. ${ }^{10}$ On basis of ab initio electronic band calculations, ${ }^{11,12}$ the interface anisotropy was attributed to the hybridization or bonding of the $3 d$ electronic states of $\mathrm{Co}$ with the conduction band of $\mathrm{Au}$. This bonding affects in a different way the electronic bands arising from Co $d$ orbitals that are either in the plane of the 


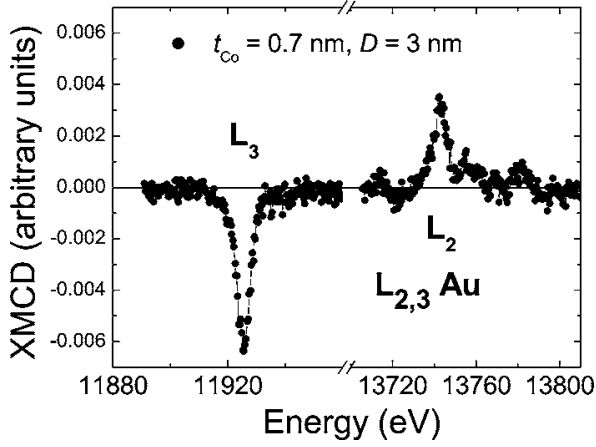

FIG. 4. X-ray magnetic circular dichroism of two samples of Au-capped $\left(t_{\mathrm{Au}}=1.5 \mathrm{~nm}\right)$ Co clusters with different average diameters. The spectra were measured at the $L_{2,3}$ edges of $\mathrm{Au}$, at $T=4.2 \mathrm{~K}$ and under a magnetic field of $1 \mathrm{~T}$.

surface or pointing perpendicular to it. The spin-orbit energies for these bands are then modified, which directly changes the magnetic anisotropy since it arises from the difference between the spin-orbit energies for spin parallel and perpendicular to the surface. This hybridization is expected to be strong in the case of $\mathrm{Au}-\mathrm{Co}$ interfaces, since the Fermi levels of both metals are rather close in energy. ${ }^{11}$

The existence of hybridization at the interface between the Co clusters and the Au overlayer is supported by x-ray absorption experiments. The spectra, plotted in Fig. 4, show XMCD signals near the $L_{2,3}$ absorption edges of Au; a clear sign that $\mathrm{Au} 3 d$ electrons become magnetically polarized by the interaction with Co. ${ }^{16}$ These experiments show that the anisotropy enhancement is accompanied by a modification of the electronic structures and magnetism of the capping metallic layer. We should mention, however, that this polarization could also mediate Ruderman-Kittel-Kasuya-Yoshida (RKKY) interactions between the nanoparticles of each layer. ${ }^{17}$ Additional interparticle interactions usually lead to larger $U .{ }^{18}$ However, we do not think that interactions are the dominant source of anisotropy in our samples. Indeed, if they were, the effect of $\mathrm{Au}$ would increase with the size of the particles, whereas the opposite is observed (see the inset of Fig. 3). In addition, interaction effects are usually suppressed by the application of relatively weak magnetic fields, ${ }^{19}$ whereas we observe a large difference in the coercive fields of capped and uncapped clusters (Fig. 2).
In conclusion, the magnetic anisotropy of nanometersized clusters can be modified by capping them by a noble metal, such as gold. This phenomenon is probably associated to the bonding of $\mathrm{Co}$ and $\mathrm{Au}$ atoms at the interface, which depends on the electronic band structures of the two metals. In principle, the same idea could apply to clusters made of other magnetic metals, thus providing a relatively simple method to tune their magnetic anisotropy. The fact that this phenomenon survives in nanoparticles offers interesting prospects for the improvement of their technological applications, in which the embedding matrix receives an active role.

The authors are grateful to A. Vaurès for help in the sample preparation. This work has been funded by Grant No. MAT02-0166 and MAT2002-04178-C04-03 from the Spanish "Ministerio de Educación y Ciencia." Two of the authors (F.B. and F.L.) acknowledge the funding from "Fundación Ramón Areces." Another author (M.J.M.) acknowledges a scholarship from CSIC.

${ }^{1}$ D. Weller and A. Moser, IEEE Trans. Magn. 35, 4423 (1999); R. L. White, J. Magn. Magn. Mater. 209, 1 (2000).

${ }^{2}$ L. Néel, Acad. Sci., Paris, C. R. 228, 664 (1949).

${ }^{3}$ F. Bødker, S. Mørup, and S. Linderoth, Phys. Rev. Lett. 72, 282 (1994). ${ }^{4}$ M. Respaud et al., Phys. Rev. B 57, 2925 (1998).

${ }^{5}$ D. A. Eastham, P. M. Denby, A. Harrison, I. W. Kirkman, and A. G. Whittaker, J. Phys.: Condens. Matter 14, 605 (2002).

${ }^{6}$ F. Luis, J. M. Torres, L. M. García, J. Bartolomé, J. Stankiewicz, F. Petroff, F. Fettar, J.-L. Maurice, and A. Vaurès, Phys. Rev. B 65, 094409 (2002).

${ }^{7}$ P. Gambardella et al., Science 300, 1130 (2003).

${ }^{8}$ P. Bruno, Phys. Rev. B 39, R865 (1989).

${ }^{9}$ F. J. A. Den Broeder, W. Hoving, and P. H. J. Bloemen, J. Magn. Magn. Mater. 93, 562 (1991).

${ }^{10}$ M. Kisielewski, A. Maziewski, M. Tekielak, A. Wawro, and L. T. Baczewski, Phys. Rev. Lett. 89, 087203 (2002).

${ }^{11}$ D. Wang, R. Wu, and A. J. Freeman, J. Magn. Magn. Mater. 129, 237 (1994).

${ }^{12}$ B. Ujfalussy, L. Szunyogh, P. Bruno, and P. Weinberger, Phys. Rev. Lett. 77, 1805 (1996).

${ }^{13}$ J. L. Maurice, J. Briático, J. Carrey, F. Petroff, L. F. Schelp, and A. Vaurès, Philos. Mag. A 79, 2921 (1999).

${ }^{14}$ W. A. Sucksmith and J. E. Thompson, Proc. R. Soc. London 225, 362 (1954).

${ }^{15}$ Y. Xie and J. A. Blackman, J. Phys.: Condens. Matter 16, 3163 (2004).

${ }^{16}$ F. Wilhelm et al., Phys. Rev. B 69, 220404(R) (2004).

${ }^{17}$ P. Bruno and C. Chappert, Phys. Rev. Lett. 67, 1602 (1991).

${ }^{18}$ F. Luis et al., Phys. Rev. Lett. 88, 217205 (2002).

${ }^{19}$ F. Luis, F. Petroff, and J. Bartolomé, J. Phys.: Condens. Matter 16, 5109 (2004). 